\title{
Telecooperation Systems in Engineering Companies Supplying the Metallurgy Industry: The Experience of the OrgTech Project
}

Giulio Iacucci, Ralph Peters

Fraunhofer Institut for Computer Graphics

Rundeturmstr. 664283 Darmstadt Germany

++496151 155209 \{giacuccilpeters\}@igd.fhg.de

Oliver Stiemerling, Volker Wulf

University of Bonn

ProSEC / Dept. of Computer Science III

Römerstr. 164, 53117 Bonn, Germany

++ 49228 73-4276 \{oslvolker\}@informatik.uni-bonn.de

\begin{abstract}
The primary goal of the ORGTech-project is to improve the cooperative work processes within and between two design offices and one steel mill through the introduction of a telecooperation system. The ORGTech approach is based on integrated organization and technology development (OTD) as a participative and evolutionary approach to support changes of work systems. In this paper we first introduce the OTD approach and explain the phases of the OrgTech Project. The outcomes of the first phase, the requirements analysis are then described and followed by an overview of co-operation tools and systems which will be tailored to the specific situation of the engineer offices and their major customer, the steel mill. We explain the next step of the project which consists of introducing the prototypes and organizational change in pilot teams, concentrating on the pilot team where groupware components are introduced.
\end{abstract}

Keywords

Telecooperation, metallurgy industry, application sharing, Computer Aided Design, participation 


\section{INTRODUCTION}

The project ORGTech (Organizational and Technical Development in the Context of the Introduction of a Telecooperation System in Small and Medium-Sized Engineering Companies) aims at supporting the cooperative work processes within and between two design offices and one steel mill. The two design offices take on subcontractual work for a large steel mill in the German Ruhr area, e.g. the construction and documentation of steel furnace components. A construction department coordinates the construction and documentation, and the contact with external offices at the steel mill. The external offices, however, complain about the specification of the construction order being vague and about the many meetings that have to take place before the project is over. The difficulty to gather the documentation about the actual state of the plants is another problem, connected with the way the drawing archive is run at the steel mill. This situation often brings inconsistencies in the final design. This problem seems to be caused by the huge number of drawings and the organization of the electronic archive, by the complexity of the construction development process, and by communication problems like the difficulty to reach the contact person.

In the next chapter we introduce the OTD approach and explain the phases of the OrgTech Project. We will then describe the outcome of the requirements analysis and explain the introduction of telecooperation tools in pilot teams.

\section{THE ORGTECH APPROACH}

The ORGTech approach is based on integrated organization and technology development (OTD) as a participative and evolutionary approach to support changes of work systems(Wulf, Rohde 1995). Within this framework we will apply instruments of organization development, work psychological guidelines, approaches to software development and tailoring in use integratedly.

The organization development process can be characterized by four steps: diagnosis, intervention planning, application of interventions and evaluation. Within this process we follow a participative approach proposed by Pieper (1988). Moreover we appliy methods and instruments of applied social science such as theory of personality, social psychology, group dynamics and organization theory (Huse, E.F. 1980). Organization development approach alone does not offer adequate criteria for the evaluation of its results.

To evaluate whether certain work systems are human centered, work psychological research seems to be very useful. Handlungsregulationstheorie (action regulation theory; cf. Hacker 1986) offers a theoretical foundation of several evaluation criteria for human-centered work systems(see also Ulich 1991). Furthermore, evolutionary and participative software development has to be supplemented by activities performed by end users or local experts of the application environment (Wulf 1994). Approaches of end users' modification of a system have been discussed under different labels (Trigg, Moran and Halasz 1987; Fischer and Girgensohn 1990; Henderson and Kyng 1991; Nardi 1993). We want 
to sum up these activities under the label of tailoring. Contrary to the development of a new system version the existing version is adapted by end users or local experts.

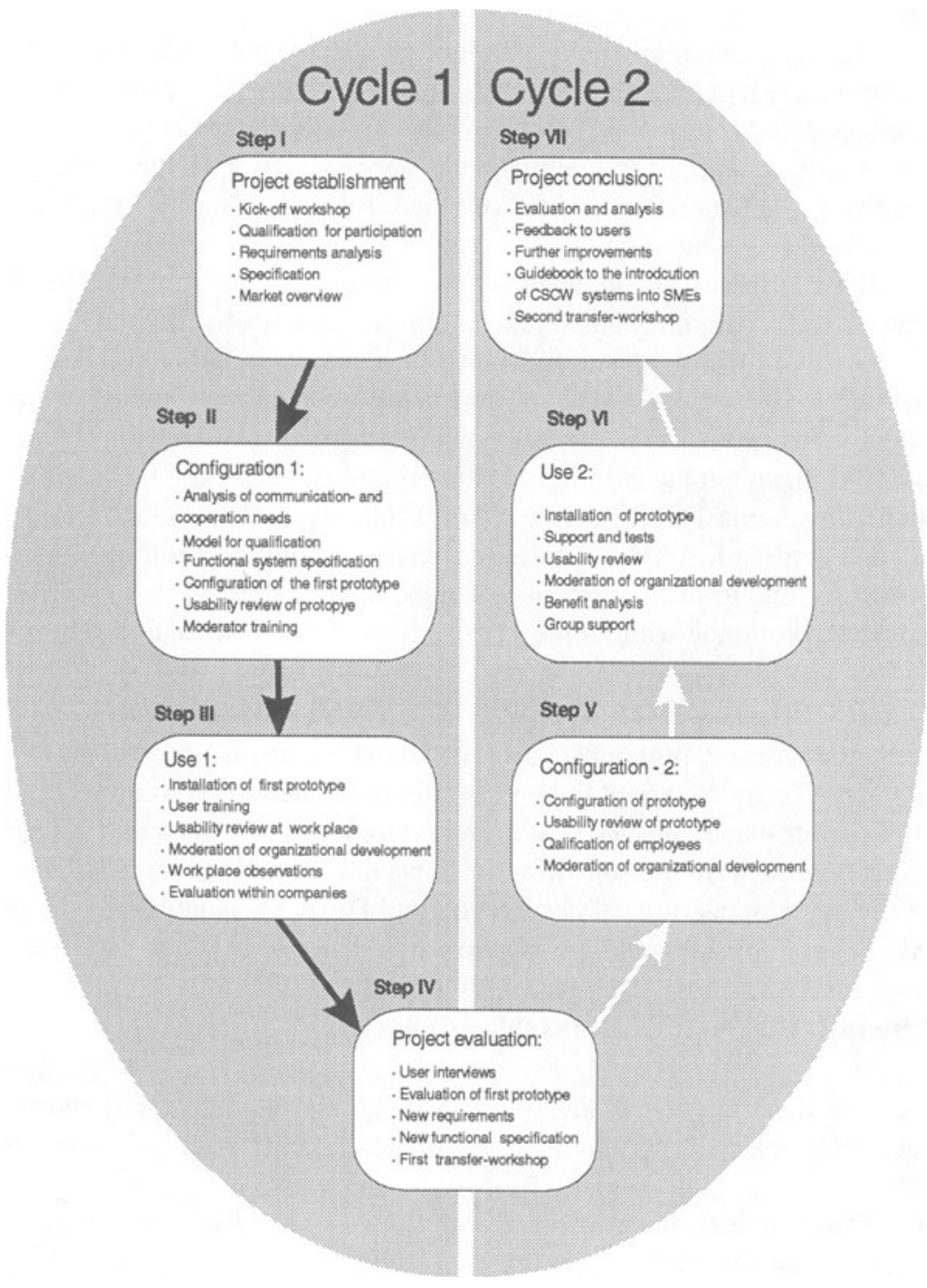

Figure 1: Model for the Org Tech process 
In the following we will describe the application of the OTD approach in the ORGTech project which concerns the introduction of a telecooperation system in the two small engineering firms, we call them here Techno and Doku, and one of their major customers the steel mill called MeltIt. OrgTech aims at supporting the cooperative work processes within and between the two engineering firms and the steel mill.

Therefore the communication patterns are analyzed and where necessary organization development is carried out. The process model for the introduction of the whole system is shown in figure 1. After the project establishment (step 1) the process mainly comprises two equivalent phases which give the project staff the opportunity to evaluate after about 1 year and use experiences from the first phase as a basis for the second.

Each phase consists of an analysis of the current situation in the fields of application, the configuration and tailoring of the technical system and - in parallel - the organization development and the qualifications of the employees involved. The second phase of the OTD process ends with another reevaluation of the integrated socio-technical work system and the feedback of the results to the users involved. At this point the members of the organizations should be able to continue the process by themselves, to perform workshops for further qualification, problem solving and conflict handling, to tailor the shared communication system according to their needs, and to restructure workgroups, if necessary. Thus, the OTD process is supposed to continue without the involvement of external change agents

\section{THE OUTCOME OF THE PROJECT ESTABLISHMENT}

We will now review problems that have emerged in the project establishment phase of ORGTech. We are at the end of this phase and have completed a kick-off workshop from which we derived an interview methodology and we interviewed our project partners. In the following lines we describe the point of view of MeltIt and of the two engineering offices Techno and Doku. We summarize the problems related to communication and information infrastructure of the three organization.

\subsection{The point of view of the steel mill MeltIt}

Only a few departments of the huge factory organization are relevant for the project. We interviewed the managers and employees of the following departments:

- Construction department, which manages the outsourcing of the construction design,

- Job planning, which receives the drawings from the construction department and plans the production,

- Mechanical workshop which produces mechanical pieces, tubes etc. to maintain the mill, 
- Systems and method department, also managing the electronic archive of the drawings used by all departments.

The construction department manages the maintenance of the different production facilities. If an operator of a production facility requires a repair service or a new part $\mathrm{s} / \mathrm{he}$ contacts the construction department. This department decides who will carry out the design and drawing, co-ordinates the process of design, and when the drawing is finished decides whether the internal workshop or an external enterprise will manufacture the part. A typical task of the construction department is to supply the external engineering offices with documentation and drawings, and to discuss the design with them. From the commission of the order until the final acceptance, the external engineer may meet quite frequently with the construction department. Sometimes an on-the-spot visit at the plant with the operator is needed, but most of the meetings take place at the construction department to discuss the drawings of the external offices.

The information infrastructure allows all the departments mentioned and the operators to access the central electronic archive of more then 100,000 drawings through a FDDI ring (100 megabit/s). The archive system allows for searching, viewing and ordering of drawings. The system allows multiple users to simultaneously open the same drawing in read only mode. A technician from the construction department and one from the job-planning department said that they used this viewing functionality to discuss design or manufacturing problems over the telephone. Such an improvisational solution of computer supported cooperative work (CSCW) has been tried out by those employees who tend to have more of an interest in such new technology. This attempt is a clear demonstration of how groupware can improve communication within MeltIt or between the construction department and the external offices.

Another relevant problem area of the steel mill is the drawing archive system. The system presents for each drawing only the latest version. If a part is altered and the new drawing has been saved, it is no longer possible to obtain the older versions of the drawings for the remaining parts of the same type. Furthermore, the key number that allows for searching the electronic archive is too unspecific. This incurs high search efforts. The jobs planning department complains that often the drawings are not ready for the workshop and some changes have to be done. Three dimensional CAD is not used at the moment but would be, in the opinion of a technician of this department, useful to communicate better with the workshop. The workers sometimes have problems reading 2D drawings, and this would improve the quality of the construction design by eliminating errors and automatically creating parts lists.

\subsection{The point of view of the engineering offices}

The engineering office Techno offers services for planning, execution, change, or capacity increase of conveying systems in heavy industry. Techno possesses various patents in this area. The office employs about a dozen engineers and has a 
Novell network with 12 CAD workstations running AutoCAD 14. One of the workstations has an ISDN connection and is used to exchange files with the steel mill. The typical design project must first retreive the drawings and documentation about the actual state. This need for drawings and documentation remains during the whole order cycle. The drawings are mostly ordered by fax from the construction department, and after printing they are piled up at MeltIt waiting to be collected. Normally every day someone from the office drives to MeltIt (about a 30 min drive away) and checks if there are drawings to collect. However, drawings still take up to one week from the day the fax order was sent. When the construction drawing is finished, it is delivered to the steel mill on a disk in an AutoCAD file and in an image file, ready to be saved in the archive. This could be done by sending the files via ISDN, but at the construction department at MeltIt only one of the managers has a PC which supports ISDN connections and the recipient of the files is usually someone else. An engineer explained that he goes to the steel mill every week, where sometimes a brief teleconference could be enough. He could also imagine discussing some of the problems with a shared viewer. The know-how and patents assure Techno other foreign customers especially overseas. The young engineer keeps contact with a customer in USA exchanging emails and faxes and talking on the phone. In this case teleconferencing has a greater potential.

The construction office Doku offers planning and documentation within the area of pipe lines and plant construction. The office has a dozen employees and one manager who is also the owner. They have a network with $10 \mathrm{CAD}$ workstations running AutoCAD 12 and 14. One PC has an ISDN connection and is used to interchange files with MeltIt. The office supplies the steel mill mostly with plant documentation. The documentation is necessary in case of failure or breakdown, but in the daily work the plant operator and the construction department of MeltIt have more important things to worry about. Doku has informal meetings with the operators more frequently then in the case of Techno, and often starts the job before the commission of the construction department takes place. The employees of Doku were rather skeptical regarding the possibilities to introduce videoconferencing. One of the technicians explained that visiting the factory is necessary and that telecooperation would not improve the situation. Because most of his communication partners on the shop floor would probably not take the time to apply the new technology. Moreover, these people need extensive explanation to be able to understand the 2D drawings. Another problem mentioned at Doku was difficulty to meet the contact person at MeltIt.

Both engineering offices would like to have permission to search and download drawings from the steel mill's archive via ISDN. On the other hand, MeltIt does not want to grant such permission for security reasons. 


\subsection{Summary of Problems}

We will now focus on communication and cooperation aspects of the processing of the construction order. We consider the following problems to have a potential solution through the introduction of information technology (IT):

The difficulty for Doku and Techno to retrieve the complete drawings and documentation for the construction. This problem is connected to the way the archive system is implemented. The database of drawings does not portray the actual state of the plants. With data sharing Techno and Doku could easily access the drawing once the permission and security issue would be properly solved.

The lack of easy ways to interchange information and documentation and to meet the contact person. The frequent interchange of drawings in file format (AutoCAD and image) is done only by Doku through ISDN. In the case of Techno the files are delivered on disks. The potential improvements concern mainly the information infrastructure. Possible solutions partially implemented are seen in the ISDN connection and internetworking.

The inconsistency in the construction drawings found in the job planning department of MeltIt. The chain from design to manufacturing involves departments and organizations who do not always cooperate together. The requests of the job planning department and the workshop are not considered at the beginning of the chain. 3D design, in the opinion of a job planner, would increase the quality of the construction drawings, facilitate the creation of parts lists and facilitate the communication and cooperation between construction engineer, job planner and workers in the workshop .

Many meetings have to take place before the final drawing is accepted. A typical construction project by Techno requires during the processing many meetings to discuss the design idea and the progress on the project with the construction department at MeltIt. In the final phase of the project many content and formal checks must take place before the drawing is accepted.

Within MeltIt employees use the viewing functionality of the archive system and telephone to discuss problems with colleagues. Groupware supplies ad hoc tools for synchronous distributed interaction. An overview follows in the next chapter (see also Peters, Kress 1997). OrgTech will concentrate on the introduction of a telecooperation system. Thus we will not deal with all of this problems. In particular the archive system will not be changed or redesigned. Furthermore, the information infrastructure will be considered to connect only the right people through a direct ISDN connection or through the Internet.

In the next chapter we will recall the functionality of groupware systems and we will give an overview of tools that could be tailored to the specific needs of Techno, Doku and MeltIt.

\section{GROUPWARE AND TELE-COOPERATION}

Computer-supported groups are, generally, project-oriented (or goal-oriented) with important tasks and tight deadlines. The group members may be present in the 
same room or they may be attending an electronic meeting at which the other members are not present in the same place. Sometimes computer-supported groups are permanent and formal groups; other situations require ad hoc groups with a finite lifetime and other kinds of properties. The group interaction might be formal or informal, spontaneous or planned, structured or unstructured. This leads to a large number of possible approaches and applications types in the area of computer support for groups (Encarnação; Noll; Peters, 1997). Although computers have been used to support team efforts, the emerging concept of computer-supported groups differs from traditional computer support concepts. Many computer systems, such as time-sharing or networked systems, are common place and only support loose aggregations of users without any support for the connection among them (cognitive, common data, simultaneous or synchronized actions). Each user is seen as a discrete unit without any semantic connection to the others. Computersupported groups introduce a new dimension and a new necessity: software designed specifically for groups - groupware.

Every phase within the cooperative working process requires the support of generic and specialized groupware applications (Peters, Kress 1995). A task management system coordinates the work of the team members and facilitates the assignment of new work items. It manages the flow of work among participants, according to a defined procedure consisting of a number of tasks (McCarthy., Bluestein 1991).

For synchronous communication within a team today's tele-cooperation systems

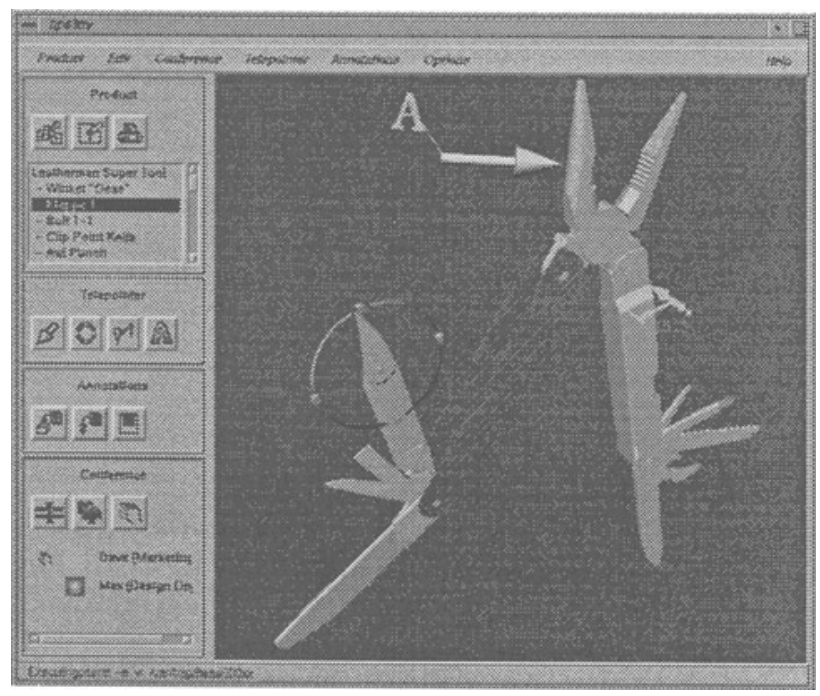

Figure2: The Share 3D Viewer

like Intel's ProShare or Microsofts NetMeeting cover the basic communication 
support with integrated audio and video connections. In these workstation integrated systems an application can be distributed to all users via a window sharing component to support shared viewing and editing. Such a joint viewing or editing tool displays the graphical output of an application simultaneously on multiple computers in a network, and thus gives all participants the same view of e.g. a document. Shared pointers are standard features and the systems come out with a modern shared whiteboard as an add on (see Peters, Kress 1997 for a detailed discussion on synchronous interaction). In order to schedule meetings with several other members of an organization electronic calendar tools can be used to determine open time slots of all participants. Advanced calendar tools consider the time slots in conjunction with the user preferences regarding time and day of the week. The Lotus' Organizer 97 and Microsoft's Outlook 97 can both be used to keep track of individual appointments and meetings.

Besides the direct tele-communication with other team-members, in a distributed CAD scenario it is also important to be able to communicate ideas by making freehand sketches and annotations. The Shared 3D Viewer (Kress 1997) is designed to support communication during the development process. The innovative 3D functionality offers engineers the proper format for the discussion of CAD data. Shared camera views, three- dimensional cursors and annotations allow the participants to discuss specific details of a product.

The user interface of the Shared 3D Viewer displays a 3D window containing the geometry of the model, and a list of the products and subproducts contained within this model with information specific to each of them (figure 2). A set of 3D cursors can be used to direct interest at specific locations on the model. Cursors can be used to mark a point, length or area of the product, and are color coded according to the user that is controlling the cursor.

Additional tools allow the measuring of lengths and angles within the threedimensional window.

\section{THE NEXT STEP: INTRODUCTION IN PILOT TEAMS}

We have now gone through the project establishment and part of the configuration (see figure 1 in chapter 2). We completed:

- the analysis of the actual state and the analysis of communication and cooperation needs,

- first qualification of the members of the three organizations with participative workshops,

- market overview of telecooperation tools, such as off the shelf products to be tailored to the specific needs for the first cycle of the project.

The next steps of the project are the configuration of prototypes of tools and systems and the introduction of these prototypes and of organizational change in pilot teams. We will describe one of the four pilot teams, where a videoconferencing system is introduced. 
The expected improvement of the introduction of groupware is to decrease misunderstandings and subsequently necessary corrections in the cooperative construction process. The introduction of the tele-cooperation system will influence the organizational structures and communication processes within and between the organizations involved and increase the product and service quality.

The introduction of groupware tools such as teleconferencing, application sharing and whiteboards are meaningful to support the discussion of construction drawings. A first scenario is within MeltIt between the construction department, job planning department and the workshop. The potential success of groupware usability in this case is assured by the examples of improvisation of CSCW described in paragraph 3.1 and by the good information infrastructure of MeltIt. Another meaningful introduction scenario is between Techno and MeltIt. In particular we found a young engineer who was interested in trying these new technology in order to avoid driving to the construction department at MeltIt. For the pilot team we have chosen this second scenario.

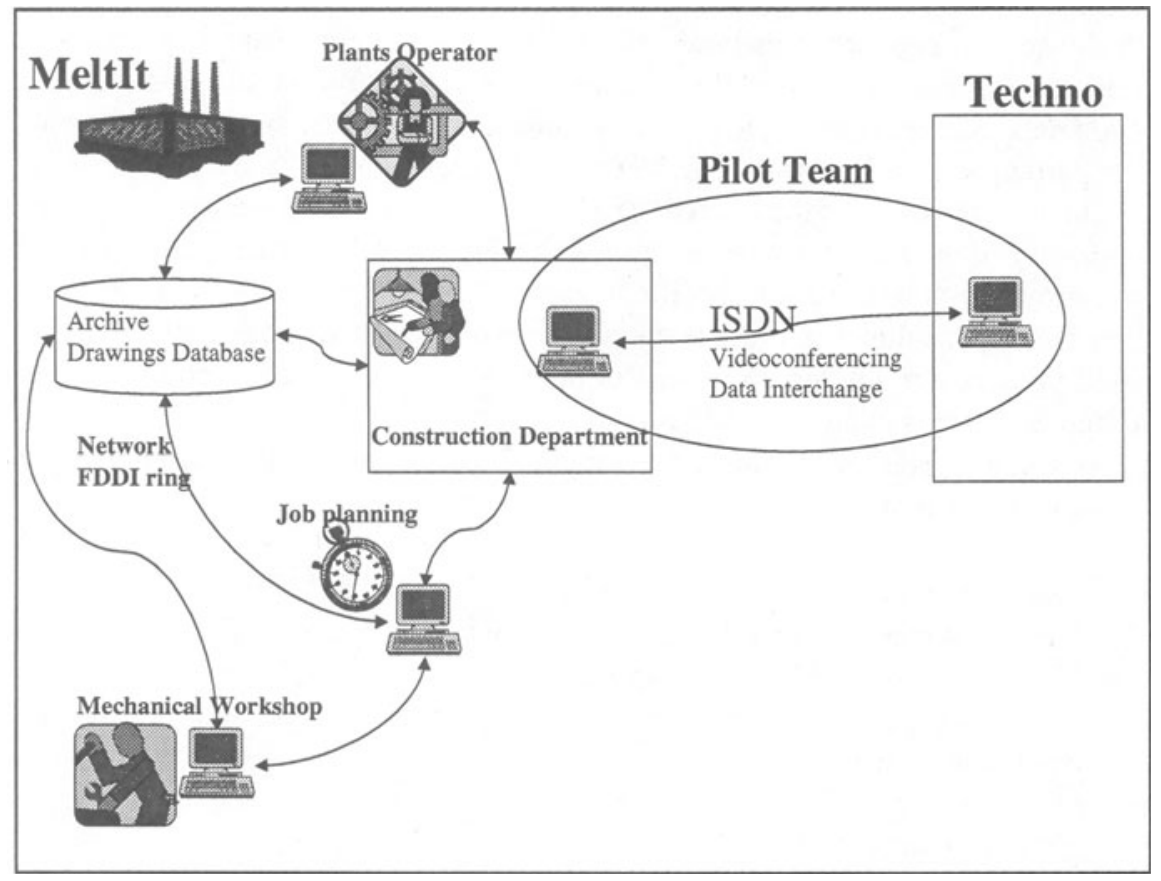

Figure 3: Information flow within MeltIt and the pilot team. 
The team is composed by one engineer from Techno and one or two contact people from the construction department. Figure 3 describes the information flow within MeltIt and the information flow of interest to the pilot team. The engineer by Techno works on a project which is followed by the members of the team at the construction department. The pilot team is therefore project oriented. The engineer at Techno and the other members of the pilot teams at the construction department will make teleconferences and use sharing applications and whiteboards. In the first cycle of the project these groupware tools are of the shelf products tailored to specific needs of the situation. ISDN allows a direct connection for the teleconferences; other groupware component like email will use the internet. The difficulty to schedule the meetings and to reach the contact person is faced with the introduction of calendar tools or email. As explained in chapter 3, these tools can be used to determine open time slots of all participants to organize the teleconferences.

We now give a short description of the goal of another of the four pilot teams, which concetrate on the introduction of three dimensional design. 3D design augments the consistency of drawings in the manufacturing and allows the easy creation of parts lists in the job planning. Moreover, three dimensional drawings are easier to read for the plant operators or workers in the workshop. The Share 3D Viewer, described in chapter 3, could support the remote discussion within MeltIt or between Techno and its customer. Three dimensional drawing are now used at MeltIt only for new construction. Doku had tried to present three dimensional drawings to its customer but with no success due to cost reasons.

In fact documentation, which is the main services offered by Doku, is a low price service and three dimensional drawings, at least at this stage, are quite expensive. The goal of this pilot team will be to understand participatively how three dimensional design can be introduced to support the processing of the construction and to augment the competitiveness of the engineering offices of Techno and Doku.

\section{CONCLUSIONS}

This paper gives an example of how the introduction of a telecooperation systems to improve competitiveness in small engineering offices can be carried out. We first described the approach of the OrgTech project and portrayed the problems that were identified in the requirements analysis. We mentioned the aim of the next step of the project which consist of the configuration and introduction of prototypes in pilot teams and in the introduction of organizational change. We gave an overview of groupware tools and concentrated on one pilot team where groupware technologies are introduced. The main objective in this case is to decrease misunderstandings and subsequent necessary corrections in the cooperative construction process. The introduction of the tele-cooperation system will influence the organizational structures and communication processes within 
and between the three organizations involved. The resulting increase in overall product and service quality will make the engineering firms more competitive.

Groupware is particularly interesting for the engineering office called Techno because of the many foreign customers. The know-how and patents of Techno assure customers especially overseas. In this case teleconferencing and sharing drawings saves time and expensive travel costs. To understand the impact of groupware technologies the participative process initiated with pilot teams is essential. As explained in chapter 2, concurrently experimenting and evaluating commercial products with the user tailored to the specific needs in the first project cycle, will be the basis for the second iteration. This iteration is particularly important in case of the introduction of groupware. As shown in several cases of introduction (see Ciborra 1996), groupware is powerful but is still unreliable and needs to be learned. The experimentation will give important indications about the usability of the tools introduced and in particular about the pilot team improvements in cooperation in compare to the traditional substitutes like Fax, telephone and meeting on the spot.

\section{REFERENCES}

Ciborra, C.U., (1996) "Groupware and Teamwork: Invisible Aid or Technical Hindrance", Wiley.

Encarnação, J.L.; Noll, S.; Peters, R. (1997): Technologies of Time- and Location Independent Telecooperation. In: Innovation and Technology - Strategies and Policies XXI. Kluwer Publication.

Fischer, G. and Girgensohn, A. (1990): End-User Modifiability in Design Environments. CHI '90, Computer Human Interaction, Conference Proceedings, Seattle, Washington, 183 - 191

Hacker, W. (1986): Arbeitspsychologie. Psychische Regulation von Arbeitstätigkeiten. VEB Deutscher Verlag der Wissenschaften, Berlin.

Henderson, A. and Kyng, M. (1991): There's No Place Like Home: Continuing Design in Use; in: Greenbaum, J.; Kyng, M.: Design at Work - Cooperative Design of Computer Artifacts, Hillsdale, 219 - 240.

Huse, E.F. (1980): Organization development, St. Paul, Min. (2nd ed.)

Kress, H.(1997): Networked Collaboration in Distributed Design Teams. In: Proceedings of the International Symposium on Global Engineering Networking (GEN '97), April 22-24, Antwerp, Belgium, pp. 235-244, HNIVerlagsschriftenreihe, Bd. 21;Rechnerintegrierte Produktion, Paderborn, 1997.

McCarthy, J.C., Bluestein, W.M.(1991): „The Computing Strategy Report: Workflow's Progress “, Forrester Research Inc., Cambridge 1991.

Nardi, B. A. (1993): A Small Matter of Programming - Perspectives on end user computing, MIT-Press, Cambridge et al.

Peters, R., Kress.H.,(1995): An approach to integrate time and locationindependent cooperation in a distributed product development process. In 
Proceedings of the International Workshop on Integrated Broadband Communications and Collaborative Work in the Automotive Industry, Trento.

Peters, R.; Kress, H., (1997): Distributed Synchronous Interaction Examined Closly. Concurrent Engineering Research and Applications. Volume 5. No.3. pp. 219-232. September 1997.

Pieper, R. (1988): Diskursive Organisationsentwicklung, de Gruyter, Berlin et al. Ulich, E.(1991): Arbeitspsychologie. Zürich: Vlg.Fachvereine; Stuttgart: Poeschel. Trigg, R. H.; Moran, T. P.and Halasz, F. G. (1987): Adaptability and tailorability in Note Cards, in: Bullinger, H.J., Shackel, B. (eds): Herman-Computer Interaction - INTERACT '87, Elsevier: Amsterdam, 723 - 728

Wulf, V. (1994): Anpaßbarkeit im Prozeß evolutionärer Systementwicklung, in: GMD-Spiegel, 24, 3, 41 - 46

Volker Wulf; Markus Rohde (1995): Towards an Integrated Organization and Technology Development; in: Proceedings of the Symposium on Designing Interactive Systems, 23. - 25.8.1995, Ann Arbor (Michigan), ACM-Press, New York 1995, S. 55 - 64 